\title{
Utilitarianism, Prioritarianism, and Intergenerational Equity: A Cake Eating Model ${ }^{*}$
}

\author{
Matthew D. Adler \\ Duke Law School
}

\author{
Nicolas Treich \\ Toulouse School of Economics, INRA
}

March 11, 2017

\begin{abstract}
We use a simple consumption model, the so-called cake eating model, to study the interaction of equity, time and risk in social decision making. Total consumption, the "cake," is uncertain. The social planner allocates consumption between two agents (representing two generations), by assigning the first a determinate amount, with the second receiving the risky remainder. We study this consumption allocation decision using three social welfare functions: utilitarianism, ex ante prioritarianism, and ex post prioritarianism. Under standard assumptions, ex ante prioritarianism allocates more consumption to the first generation than utilitarianism. Thus, a concern for equity, in the ex ante prioritarian sense, means less concern for the risky future. By contrast, ex post prioritarianism normally chooses less consumption for the first generation than utilitarianism. We discuss the robustness of these optimal consumption allocations to learning and
\end{abstract}

${ }^{*}$ We thank the associate editor and two anonymous referees for useful comments. We also thank conference participants at WCERE 2014, EGRIE 2014, HECER (Helsinki), NHH (Bergen), and Grantham Institute (London) for their comments. Nicolas Treich acknowledges funding from the European Union Seventh Framework Programme (FP72007-2013) under grant agreement $\mathrm{n}^{\circ}$ [266992] entitled GLOBAL-IQ and the chair "Finance Durable et Investissement Responsable" (FDIR). Corresponding author: Nicolas Treich. Email: ntreich@toulouse.inra.fr 
to more complicated social welfare functions.

Key words: Equity, utilitarianism, prioritarianism, risk aversion, discounting, climate change.

JEL: D81, I31, E21 


\section{Introduction}

\subsection{The general motivation}

The issue of intergenerational justice is central for many risk policies. Consider for instance climate change. A more stringent climate policy today reduces the consumption of the current generation but also reduces the risks borne by future generations. This is one example of many policies - e.g., nuclear waste management, sustainable agriculture policy - in which policy makers must trade the cost in terms of a sure reduction in current consumption against the benefit in terms of a future risk reduction. This trade-off is complex because it mixes the dimensions of equity, time and risk. It raises the fundamental problem of the optimal intertemporal allocation of risky aggregate consumption.

The object of this paper is to study this problem using a "prioritarian" approach. Formally, we consider a prioritarian social welfare function (SWF): one that takes the form $\sum_{i} g\left(u_{i}\right)$, with $u_{i}$ the utility of agent $i$ (a measure of her wellbeing), and $g($.$) a strictly increasing and concave function. We are$ especially interested in the difference between a prioritarian approach and a standard utilitarian approach captured by a simple additive SWF of the form $\sum_{i} u_{i}$. This comparison allows us to explore how equity considerations affect intertemporal consumption allocation under risk.

\subsection{The prioritarian approach}

The concept of "prioritarianism" originates in contemporary political philosophy (Parfit 1991, Nagel 1995). The key idea is to give greater weight to wellbeing changes affecting worse-off agents. This idea is captured, axiomatically, in the Pigou-Dalton principle, otherwise known as the "principle of transfers". ${ }^{1}$ The Pigou-Dalton principle is the critical axiomatic difference between prioritarianism and utilitarianism. Both SWFs satisfy axioms of Pareto superiority, anonymity, separability, and continuity $;^{2}$ but utilitarian-

\footnotetext{
${ }^{1}$ The principle can be stated as follows. If agent $j$ is better off than agent $k$, then a pure, non-rank switching transfer of well-being between them (so that $j$ goes from $u_{j}$ to $u_{j}-\Delta u, k$ from $u_{k}$ to $u_{k}+\Delta u$, with $\Delta u>0, u_{j}-\Delta u \geq u_{k}+\Delta u$ ), with everyone else unaffected, is an ethical improvement.

${ }^{2}$ See, for instance, Adler (2012). Note that there also exists an axiomatic foundation to prioritarianism based on an extension of Harsanyi's utilitarian impartial observer theorem, and coined "generalized utilitarianism" (Grant et al. 2010). For a criticism of
} 
ism violates the Pigou-Dalton principle since a pure transfer leaves unchanged the sum total of wellbeing. In economics, prioritarian SWFs have been used in social choice and optimal taxation literatures (Sen 1970, Kaplow 2008) as well as in policy evaluation when "distributional weights" capture the nonlinearity in both the utility and the social welfare functions (Drèze and Stern 1987, Johansson-Stenman 2005, Adler 2016a).

The central difference between utilitarianism and prioritarianism is that the latter cares both about total wellbeing and about the distribution of wellbeing. Although utilitarianism does take account of the distribution of income (given the declining marginal utility of income), it is insensitive to the distribution of wellbeing itself. And many have criticized utilitarianism on these grounds (Parfit 1991, Tungodden 2003, Holtug 2010, Porter 2012). The concept of prioritarianism is now well understood not only in philosophy, but in social choice theory and theoretical welfare economics. Indeed, the axiomatic characterization of prioritarianism comes from economic theory. However, the vast majority of work that uses the SWF construct implements a utilitarian SWF. For example, the debates about climate policy have largely assumed a utilitarian SWF (Stern 2007, Dasgupta 2008, Nordhaus 2008, Weitzman 2008, Gollier 2012), disagreeing about parameters of the utility function and the appropriateness of adding a time discount factor, but not about the basic utilitarian formula.

From a pure theoretical perspective, utilitarianism and prioritarianism are formally similar in a riskless world. ${ }^{3}$ However, the similarity does not hold under risk. And, as we said above, for real-world policy applications like climate change, it is important to consider risk - where the social planner does not know for certain what distribution of wellbeing will result from the various policy choices available to him. In the context of normative risky choice, an important distinction is between ex ante and ex post prioritarianism. The ex ante prioritarian social planner applies the $g($.$) function$

prioritarianism, see for instance Harsanyi (1975) and Broome (1991).

${ }^{3}$ It is obvious indeed that the prioritarian SWF can be written $\sum_{i} g\left(u_{i}\right)=\sum_{i} v_{i}$ where $v_{i}=g\left(u_{i}\right)$, and thus has the utilitarian form. We should nevertheless add an important word of caution here. Indeed, the choice of a specific $g($.$) may introduce "constraints"$ on the choice of $u$. This is because prioritarianism requires a more precise scaling of the utility function than utilitarianism, and additional normative choices need to be made to achieve such scaling. Besides, prioritarianism also requires additional normative choices to model (e.g.) intra-generational equity or variable population. As a result, prioritarianism can differ substantially from utilitarianism in such richer settings even when the world is riskless. These issues are discussed at length in Adler and Treich (2015). 
to individuals' expected utilities, and maximizes the sum of concavely transformed expected utilities; while the ex post prioritarian social planner applies the $g($.$) function to individuals' final utilities, and maximizes the expected$ sum of concavely transformed final (realized) utilities. As a result, the ex post prioritarian social planner cares about the difference in realized utilities ex post, once the risk is resolved; while the ex ante prioritarian social planner cares about the difference in expected utilities ex ante, before the risk is resolved. The choice between the ex ante and the ex post criterion is linked to the well known distinction between equality of opportunities and equality of outcomes. This choice has been extensively discussed in social choice (Diamond 1967, Broome 1984, Fleurbaey 2010, Adler 2012, Fleurbaey and Bovens 2012, Mongin and Pivato 2016). In this paper, we remain agnostic about whether one should use one or the other criterion. Instead, we study the implications of this normative choice for intertemporal consumption under risk. In turn, we emphasize the importance and richness of the ex ante/ex post distinction for a large class of policies commonly studied by economists.

\subsection{The main contribution}

We consider a simple consumption model which has the following interpretation. A social planner must split a cake among agents who arrive sequentially (e.g., among successive generations). Under certainty, the problem is easy, and the cake is equally shared when the agents are identical. But the problem is that the size of the cake is unknown. If the social planner is prioritarian rather than utilitarian, should he give more or less of the cake to the first agent, given that the remaining portion of the cake is unknown?

Although our consumption model is quite simple, it is - we believe - a fruitful model for exploring the differences between utilitarianism and prioritarianism. Indeed, this model has been a standard tool to explore the interaction between risk and time, for instance in the literature on climate change, social discounting or non-renewable resources. In this paper, we add the third dimension of equity, and consider how the optimal consumption allocationsfrom an ex ante prioritarian or ex post prioritarian perspective - are affected by the interaction of equity, time and risk. This three-way interaction depends, in subtle ways that we describe, on the functional form of both $u($.$) ,$ the shape of the individual utility function, and $g($.$) , the shape of the SWF.$ We assume throughout that the utility function $u($.$) is the same for every$ 
agent - thereby avoiding the thorny question of how different wellbeing measures might be compared across agents (Arrow 1951, Dhillon and Mertens 1999). This assumption is prevalent in the applied literature using SWFs, and provides a natural starting point for our analysis. ${ }^{4}$

The main result of the paper is that the optimal consumption rule depends sensitively on whether one uses an ex ante or an ex post prioritarian approach. We show that the social planner should give more of the cake to the first agent under ex ante prioritarianism than under utilitarianism, but less under ex post prioritarianism than under utilitarianism. We also show that this result is robust to a situation in which the social planner learns the size of the cake after the first consumption allocation decision has been made. Finally, we discuss whether this result is robust to more general forms of prioritarianism. In particular, we derive a simple condition regarding the comparison of "transformed" ex post prioritarianism and utilitarianism. We also consider Fleurbaey (2010)'s equally distributed equivalent (EDE) function, an important class of transformed ex post criteria, and exhibit a case where the social decisions under EDE are identical to those made under utilitarianism.

\subsection{The related literature}

Our basic setting is formally similar to a standard precautionary savings model. As many scholars before, we consider the simplest two-period version of this model (Leland 1968, Sandmo 1970, Drèze and Modigliani 1972, Kimball 1990, Gollier 2001), and focus on the comparative statics analysis of different SWFs. We use this model as a common device for exploring how optimal consumption allocation is affected by the interaction between risk and time. This device has typically been used to think about climate discounting under growth risk (Gollier 2003, Weitzman 2009, Millner 2013). Interestingly, the climate economics literature has been recently extended to non-utilitarian SWFs in a multi-periodic setting (Ha Duong and Treich 2004, Roemer 2008, Traeger 2012, Dietz and Asheim 2012, Jensen and Traeger 2014, Fleurbaey and Zuber 2015).

\footnotetext{
${ }^{4}$ In a related paper, Adler and Treich (2015) review some distinctive normative choices that are required for the prioritarian approach, including the issue of the specification of a ratio scale for wellbeing (if the prioritarian SWF takes the standard "Atkinson" form), and the determination of the degree of concavity of the $g($.$) function (i.e., the degree of$ inequality aversion).
} 
The distinction between the ex ante and the ex post approach has been explored by Ulph (1982), Fleurbaey and Bovens (2012) and Adler, Hammitt and Treich (2014), all in the context of mortality risk policies. Fleurbaey and Zuber (2015) adopt an EDE ex post approach and examine the optimal social discount rate. Ferranna (2016a, 2016b) also uses the EDE approach to compute the social cost of financial and mortality risk and the social cost of carbon. Bommier, Lanz and Zuber (2015) consider an ex post approach (i.e., risk-sensitive preferences, Bommier and Zuber 2008) and study a model of economic growth, stock pollutant and endogenous risk of catastrophe. All these papers contrast the ex post approach and the classical utilitarian approach. However, we are not aware of a general result in the literature that stresses the opposing implications of the ex ante and ex post approaches as compared to utilitarianism, as we find in this paper. For instance, Ferranna (2016a) shows that the social cost of risk tends to be lower under utilitarianism than under both the ex post EDE approach and the ex ante approach she considers (i.e., the equality of prospects, Grant et al. 2010).

\section{A simple consumption model}

We consider the problem of a social planner who must split a cake between two agents. The size of the cake $\widetilde{w}$ is unknown. We assume that this size is finite and strictly positive, and we denote $w_{\text {inf }}>0$ the smallest realization of $\widetilde{w}$. We also assume that the two agents have the same utility function $u$, with $u$ strictly increasing, strictly concave and thrice differentiable. That is, the only difference between agent 1 and agent 2 is the sequence and the risk allocation. Agent 1 arrives first, and receives a riskless portion of the cake, c. Agent 2 arrives second, and receives the remaining risky portion of the cake, $\widetilde{w}-c$. Note that, since the utility functions are strictly increasing, it is always optimal that the cake is fully consumed.

The main objective of our analysis is to compare the levels of consumption of the cake by the first agent across three different social planner's objectives, namely utilitarianism, ex ante prioritarianism and ex post prioritarianism. To be clear, we stress that the agents do not make a choice in this model (except the trivial one of consuming all the cake that is given to them by the social planner). Our analysis thus explores how the preferences of the social planner toward equity affect consumption allocation decisions under risk. Under utilitarianism, the optimal first agent's consumption, denoted 
$c^{U}$, is simply defined by

$$
c^{U}=\arg \max _{c} u(c)+E u(\widetilde{w}-c) .
$$

Before solving the model, we make two remarks. First, our cake eating problem admits several interpretations. A natural interpretation is when $\widetilde{w}$ is a non-renewable resource of unknown size that can be consumed over time by successive generations (Kemp 1976). Another interpretation is that of a social planner who decides how much the first generation should save for a second generation under uncertain intertemporal aggregate wealth $\widetilde{w}$ (Gollier 2003). ${ }^{5}$ Finally, we stress that our model is formally similar to a precautionary savings model. Nevertheless, we do not adopt that single-agent interpretation in the following because we are interested in the social planner's allocation problem between several agents. The second remark is that in the model above we have implicitly assumed that it is never optimal that the cake is fully consumed by the first agent, i.e. $c^{U}<w_{\text {inf }}{ }^{6}$ This always holds whenever $u^{\prime}\left(w_{\text {inf }}\right)<E u^{\prime}\left(\widetilde{w}-w_{\text {inf }}\right)$, a condition which depends on the probability distribution over the size of the cake and/or on the utility function. Note that the assumption $u^{\prime}(0)=+\infty$ is sufficient for this condition to hold. We recognize however that this last assumption on the utility function may be too strong in general.

The first order condition (FOC) of program (1) gives ${ }^{7}$

$$
u^{\prime}\left(c^{U}\right)-E u^{\prime}\left(\widetilde{w}-c^{U}\right)=0 .
$$

\footnotetext{
${ }^{5}$ It is also possible to interpret the model so that there is no temporal dimension; instead, the two agents are contemporaneous. Consider for instance two agents living on two different islands. The problem of the social planner is that of choosing how much food to deliver to the first island before going to the second island. Relatedly, consider the "sharing a river" problem with upstream and downstream agents (Ambec and Sprumont 2002). The social planner's problem then is to decide water allocation schemes when downstream agents face a risk that upstream agents do not face. Note however that such interpretations should explain why the social planner cannot implement risk-sharing schemes between the contemporaneous agents.

${ }^{6}$ Interestingly, the early papers on non-renewable resource consumption under uncertainty explored this possibility of a "premature exhaustion" of the cake (Kemp 1976, Loury 1978, Gilbert 1979). In these papers, at each moment the social planner updates his beliefs about the size of the cake conditional on the observation that this size can be no less than what has been effectively consumed.

${ }^{7}$ Second order conditions are satisfied throughout the paper, except when explicitly mentioned.
} 
It is easy to see that agent 1's consumption is reduced under risk, i.e. $c^{U} \leq$ $\frac{E \widetilde{w}}{2}$, if and only if (iff) $u$ is "prudent", i.e. $u^{\prime \prime \prime} \geq 0$ (Leland 1968, Kimball 1990). Indeed, that result holds iff $E u^{\prime}(\widetilde{w}-c) \geq u^{\prime}(E \widetilde{w}-c)$ for all $\widetilde{w}$, namely iff marginal utility is convex by Jensen's inequality. In other words, under prudence, the marginal utility of wealth is higher under risk, and thus it makes sense to transfer more wealth to the second agent when this latter agent faces a risk. Note that the restriction $u^{\prime \prime \prime}>0$ is necessary for the common decreasing absolute risk aversion (DARA) hypothesis, and is usually accepted in the risk theory literature (Gollier 2001). ${ }^{8}$

\section{Ex ante prioritarianism}

Under ex ante prioritarianism (EAP), the optimal consumption of the first agent is defined by

$$
c^{E A P}=\arg \max _{c} g(u(c))+g(E u(\widetilde{w}-c)),
$$

where $g$ is strictly increasing, strictly concave and thrice differentiable. Note that the social planner maximizes the sum of transformed expected utilities, consistent with an ex ante approach. The optimal level of consumption is characterized by the following FOC:

$$
f\left(c^{E A P}\right) \equiv g^{\prime}\left(u\left(c^{E A P}\right)\right) u^{\prime}\left(c^{E A P}\right)-g^{\prime}\left(E u\left(\widetilde{w}-c^{E A P}\right)\right) E u^{\prime}\left(\widetilde{w}-c^{E A P}\right)=0 .
$$

The first agent thus consumes more under EAP than under utilitarianism, i.e. $c^{E A P} \geq c^{U}$, when $f\left(c^{U}\right) \geq 0$, which by using (2) holds iff

$$
u\left(c^{U}\right) \leq E u\left(\widetilde{w}-c^{U}\right) .
$$

\footnotetext{
${ }^{8}$ It should be noted that the DARA property is usually extracted from individual choices over lotteries, for instance by examining precautionary savings choices. But in our model, as we emphasized above, agents make no choices. Rather, utility numbers are an input into the social planner's SWF, which he uses to make allocation decisions. So why assume that the utility function, $u($.$) , should satisfy DARA? The answer is that a$ plausible $u($.$) should respect preferences. It should assign a higher expected utility number$ to one consumption lottery for a given individual than a second consumption lottery iff the individual herself would prefer the first lottery. This implies that $u($.$) must be an$ affine transformation of the individual's own utility function - the one that guides her own choices (Adler 2016b). But DARA is preserved under affine transformations. Thus the evidence of DARA in individual choices implies that the $u($.$) function used by the social$ planner in our model should also be DARA.
} 
This leads to the following result. ${ }^{9}$

Proposition 1 The first agent consumes more under EAP than under utilitarianism iff $u$ is $D A R A$, i.e.

$$
\frac{u^{\prime \prime \prime}(w)}{-u^{\prime \prime}(w)} \geq \frac{-u^{\prime \prime}(w)}{u^{\prime}(w)}
$$

Proof: Under DARA, $-u^{\prime}$ is more risk averse than $u$. Namely, we have $u=\phi\left(-u^{\prime}\right)$ with $\phi$ strictly increasing and convex. This leads to

$$
\begin{aligned}
E u\left(\widetilde{w}-c^{U}\right) & =E \phi\left(-u^{\prime}\left(\widetilde{w}-c^{U}\right)\right) \\
& \geq \phi\left(-E u^{\prime}\left(\widetilde{w}-c^{U}\right)\right) \\
& =\phi\left(-u^{\prime}\left(c^{U}\right)\right) \\
& =u\left(c^{U}\right),
\end{aligned}
$$

which proves the inequality (5) above. We now show the necessity. If $u$ is not DARA, then $\phi$ is locally concave, and the above inequality can be reversed for a well chosen $\widetilde{w}$. Therefore, the first agent's consumption under EAP could be made lower than under utilitarianism. Q.E.D

The intuition for this result may be presented as follows. Assuming utilitarianism, under DARA (and thus under prudence) the reduction in agent 1's consumption due to risk implies that agent 2's future expected utility is greater than agent 1's utility (see (5)). This in turn gives the ex ante prioritarian social planner an incentive to increase agent 1's consumption, thereby reducing the difference between agent 1 and agent 2's (expected) utilities. Hence, this result shows that under a standard assumption on the utility function, prioritarianism leads to more, and not less, consumption of the first agent. ${ }^{10}$ With the intergenerational justice issue in mind, this result indicates that a concern for equity (in the EAP sense) means less concern for

\footnotetext{
${ }^{9}$ All the Propositions in this paper should be understood as stating results which hold true for every level of wealth $w$. This is a key assumption for the necessary conditions stated in the Propositions. Moreover, unless otherwise noted, "more" means "weakly more" and "less" means "weakly less."

${ }^{10}$ Since there is more consumption under EAP than under utilitarianism, one may wonder whether it is possible that there is more consumption under EAP than under certainty (under either utilitarianism or prioritarianism). It is straightforward to show that this is never the case under $u^{\prime \prime \prime} \geq 0$.
} 
the risky future. Notice here that ex ante fairness is often viewed as socially desirable in the literature (Diamond 1967, Epstein and Segal 1991). Our model thus maybe illustrates a surprising implication of this view.

Note that under constant absolute risk aversion (CARA), we have $u\left(c^{U}\right)=$ $E u\left(\widetilde{w}-c^{U}\right)$ leading to $c^{U}=c^{E A P}$ and thus to $u\left(c^{E A P}\right)=E u\left(\widetilde{w}-c^{E A P}\right)$. Namely, under the common CARA utility function, the (expected) utilities of the two agents are equal both under utilitarianism and under prioritarianism.

Note also that in the model the risk is concentrated on agent 2, and that the agent 1 faces no risk. The model is thus specific as it assumes an asymmetric allocation of risk between the agents. This limitation may be partially overcome. Indeed it is well known that DARA is preserved by the introduction of an independent background risk (Gollier 2001, p.116). That is, the utility function $u()=.E u_{0}(.+\widetilde{\varepsilon})$ with $E \widetilde{\varepsilon}=0$ is DARA when the primitive utility function $u_{0}$ is DARA. Hence, the result of Proposition 1 is preserved if DARA agents $u_{0}$ would face a background risk $\widetilde{\varepsilon}$ (independent of $\widetilde{w}$ ). This does not mean however that the result would be preserved if the agents would each face different background risks. In fact, the general problem of how to allocate consumption between agents who differ in the risks they face is a much more general and complex problem, and is not further pursued here. ${ }^{11}$

We finally add a comment about the scaling of the vNM utility function $u($.$) . Under expected utility, it is well known that the utility function is$ unique up to a positive affine transformation. Under utilitarianism (1), a change from $u($.$) to some other u^{*}()=.a u()+$.$b with a>0$ does not change consumption. However, under prioritarianism, this is no longer true. For a given $g($.$) in the SWF, consumption under EAP may differ after such$ an affine transformation. Yet, since DARA is preserved under any affine transformation, the result of Proposition 1 is also preserved. Namely, for any given $g($.$) , and for any given u($.$) that is DARA, agent 1's consumption$ under EAP is greater than under utilitarianism both under $u($.$) and for every$ positive affine rescaling of $u($.$) .$

\footnotetext{
${ }^{11}$ To give a flavor of the complexity (only for the utilitarian case), let the following more general problem of providing a transfer $c$ between two agents facing different risks: $\max _{c} E u\left(\widetilde{w}_{1}+c\right)+E u\left(\widetilde{w}_{2}-c\right)$. The transfer $c$ is positive iff $E u^{\prime}\left(\widetilde{w}_{1}\right) \geq E u^{\prime}\left(\widetilde{w}_{2}\right)$. Then if $\widetilde{w}_{1}$ dominates $\widetilde{w}_{2}$ via $M$ th order stochastic dominance this holds whenever the $(M+1)$ th derivative of $u$ is positive. See Eeckhoudt and Schlesinger (2006) for an introduction to the literature on higher-order derivatives of the utility function.
} 


\section{Ex post prioritarianism}

Under ex post prioritarianism (EPP), the optimal consumption of the first agent is defined by

$$
c^{E P P}=\arg \max _{c} g(u(c))+E g(u(\widetilde{w}-c)) .
$$

Note that the social planner now maximizes the expectation of transformed utilities, consistent with an ex post approach. ${ }^{12}$ Let us clarify here how we obtain this objective. Assume that $\widetilde{w}=w_{s}$ in state $s$. The EPP social planner then reaches welfare $g(u(c))+g\left(u\left(w_{s}-c\right)\right)$ in that state. Before the realization of uncertainty, the expected welfare under EPP is thus $E\{g(u(c))+g(u(\widetilde{w}-$ $c))\}$, which is equivalent to (6) because the wellbeing of the first agent, i.e. $u(c)$, is certain.

The FOC is given by

$$
k\left(c^{E P P}\right) \equiv g^{\prime}\left(u\left(c^{E P P}\right)\right) u^{\prime}\left(c^{E P P}\right)-E g^{\prime}\left(u\left(\widetilde{w}-c^{E P P}\right)\right) u^{\prime}\left(\widetilde{w}-c^{E P P}\right)=0 .
$$

The first agent consumes less under EPP than under EAP iff $k\left(c^{E A P}\right) \leq 0$. In the following Proposition, we derive a sufficient condition for this inequality.

Proposition 2 The first agent consumes less under EPP than under EAP when $g^{\prime \prime \prime} \geq 0$.

Proof: Let $\widetilde{w}-c^{E A P} \equiv \widetilde{z}$. Then observe that $k\left(c^{E A P}\right) \leq 0$ iff

$$
g^{\prime}(E u(\widetilde{z})) E u^{\prime}(\widetilde{z}) \leq E g^{\prime}(u(\widetilde{z})) u^{\prime}(\widetilde{z}) .
$$

Observe now that $E g^{\prime}(u(\widetilde{z})) u^{\prime}(\widetilde{z})=E g^{\prime}(u(\widetilde{z})) E u^{\prime}(\widetilde{z})+\operatorname{Cov}\left(g^{\prime}(u(\widetilde{z})), u^{\prime}(\widetilde{z})\right)$, and since $g^{\prime}(u(z))$ and $u^{\prime}(z)$ are both decreasing in $z$, the covariance term is positive. Therefore the result holds if $g^{\prime}(E u(\widetilde{z})) \leq E g^{\prime}(u(\widetilde{z}))$, which is the case iff $g^{\prime \prime \prime} \geq 0$ by Jensen inequality. Q.E.D.

\footnotetext{
${ }^{12}$ Since EAP and EPP represent two different normative criteria, one may wonder why we keep the same function $g$ for both criteria. A simple reason is that, under certainty, EAP and EPP only agree with the same $g$. We note, however, that the main result of the paper, i.e., $c^{E A P} \geq c^{U} \geq c^{E P P}$, still holds for different $g$ functions for EAP and EPP (say, $g_{E A P}$ and $\left.g_{E P P}\right)$ under the conditions presented in our Propositions applied respectively to each function.
} 
Note that the result only requires a restriction on the third derivative of the function $g($.$) , with no restriction on u($.$) . Thus, this result is not affected$ by the scaling of the utility function.

Is the restriction $g^{\prime \prime \prime} \geq 0$ plausible? At a minimum, this restriction is surely more plausible than the opposite $g^{\prime \prime \prime}<0$. In fact, it can be shown that if $g^{\prime}(u)>0$ and $g^{\prime \prime}(u)<0$ and if $g^{\prime \prime \prime}(u)$ has the same sign for all $u>0$, then it must be that $g^{\prime \prime \prime}(u)>0$ (Menegatti 2001). Indeed a positive, strictly decreasing and concave $g^{\prime}$ would have to cross the origin at some point (thus contradicting $g^{\prime}>0$ ), as illustrated in Figure 1. Note that the standard Atkinsonian function, i.e. $g(u)=(1-m)^{-1} u^{1-m}$ with $u>0$ and $m>0$ (and $g(u)=\log u$ for $m=1$ ), displays $g^{\prime \prime \prime}>0$. Another standard prioritarian transformation function is the negative exponential function, i.e. $g(u)=-e^{-u}$, which also displays $g^{\prime \prime \prime}>0$.

\section{$<$ INSERT FIGURE $1>$}

Thus, under commonly used SWFs, the first agent consumes less under EPP than under EAP. The next objective is to examine whether there could be also less consumption under EPP than under utilitarianism. We know the answer in the CARA case. We saw above that if $u$ has the CARA form, agent 1's consumption under EAP is equal to that under utilitarianism. Therefore Proposition 2 indicates that the first agent's consumption under EPP is also lower than under utilitarianism under CARA when $g^{\prime \prime \prime} \geq 0$. But we would want to sign the comparison between EAP and utilitarianism in the general case. The answer is given in the following Proposition.

Proposition 3 The first agent consumes less under EPP than under utilitarianism iff

$$
\frac{u^{\prime \prime \prime}(w)}{-u^{\prime \prime}(w)} \leq 3 \frac{-u^{\prime \prime}(w)}{u^{\prime}(w)}+\left\{\frac{g^{\prime \prime \prime}(u(w))}{-g^{\prime \prime}(u(w))}\right\} u^{\prime}(w) .
$$

Proof: Let us define $v()=.g(u()$.$) . We want to examine under which$ conditions we have: $u^{\prime}\left(c^{U}\right)-E u^{\prime}\left(\widetilde{w}-c^{U}\right)=0$ implies $v^{\prime}\left(c^{U}\right)-E v^{\prime}\left(\widetilde{w}-c^{U}\right) \leq 0$. Now let $-v^{\prime}()=.\varphi\left(-u^{\prime}().\right)$ with $\varphi$ strictly increasing and concave. Then

$$
\begin{aligned}
-E v^{\prime}\left(\widetilde{w}-c^{U}\right) & =E \varphi\left(-u^{\prime}\left(\widetilde{w}-c^{U}\right)\right) \\
& \leq \varphi\left(-E u^{\prime}\left(\widetilde{w}-c^{U}\right)\right) \\
& =\varphi\left(-u^{\prime}\left(c^{U}\right)\right) \\
& =-v^{\prime}\left(c^{U}\right) .
\end{aligned}
$$


Conversely, if $\varphi$ is locally convex, then it is possible to find a well chosen $\widetilde{w}$ so that the inequality above is reversed. Therefore the necessary and sufficient condition is that $-v^{\prime}$ is more concave than $-u^{\prime}$, or $\frac{v^{\prime \prime \prime}}{-v^{\prime \prime}} \geq \frac{u^{\prime \prime \prime}}{-u^{\prime \prime}}$ given that $v$ is itself more concave than $u$. This condition is provided in the theorem 3.4 in Eeckhoudt and Schlesinger (1994), which yields (8). Q.E.D

Why is condition (8) so complex? Denoting $v()=.g(u()$.$) , the proof$ shows that the comparison between EPP and utilitarianism depends on how a change in preference from $u$ to $v$ affects consumption. Hence, comparing consumption under EPP and utilitarianism is formally similar to analyzing the effect of more risk aversion in a precautionary savings model. More precisely, it depends on whether more risk aversion, i.e. $\frac{-u^{\prime \prime}}{u^{\prime}} \leq \frac{-v^{\prime \prime}}{v^{\prime}}$, leads to more prudence, i.e. $\frac{u^{\prime \prime \prime}}{-u^{\prime \prime}} \leq \frac{v^{\prime \prime \prime}}{-v^{\prime \prime}}$. This last implication explains why condition (8) involves the third derivatives of both $u$ and $g$ as one needs to compute $v^{\prime \prime \prime}$. Although it sounds intuitive that a more risk averse agent should be more prudent, this is not always the case (Eeckhoudt and Schlesinger 1994). For instance, one may change the degree of risk aversion of a quadratic utility function without affecting the degree of prudence. ${ }^{13}$ Furthermore, we stress here that changing $g($.$) in (6) is not a proper way to study the effect of more$ risk aversion in the precautionary savings model (Bommier, Chassagnon and Le Grand 2012). Indeed, this change not only affects risk aversion, but also intertemporal substitution motives. In other words, this change affects ordinal preferences over certain prospects, and is not appropriate to capture a "pure" change in risk preferences in intertemporal expected utility models. ${ }^{14}$

We now investigate whether the condition (8) is satisfied for most commonly used utility functions and SWFs. We consider the prevalent Atkinsonian SWF, i.e. $g(u)=(1-m)^{-1} u^{1-m}$ with $u>0$ and $m>0$. In that case,

\footnotetext{
${ }^{13}$ Moreover, here is an example where the condition (8) does not hold for some wealth levels. Take $g(u)=-e^{-u}$ and $u(w)=(1-\gamma)^{-1} w^{1-\gamma}$, then the condition is violated iff wealth is below $\widehat{w}=(1-2 \gamma)^{\frac{1}{1-\gamma}}$.

${ }^{14}$ As an attempt to address this concern, an early approach was proposed by Khilstrom and Mirman (1974). They consider a model of the form $\left.\max _{c} g^{-1}(E g[u(c)+u(\widetilde{w}-c))]\right)$. In this model, more risk aversion correponds to a more concave $g$ and decreases first period consumption (for "small risks", see Drèze and Modigliani 1972, Bommier, Chassagnon and Le Grand 2012). A well known alternative is based on so-called "recursive" preferences (Selden 1978, Epstein and Zin 1989), leading to the objective: $\max _{c} u(c)+u\left(g^{-1}(E g((\widetilde{w}-\right.$ $c))$ ). A more concave $g($.$) is also interpreted as more risk aversion, and it reduces current$ consumption given some specific technical restrictions on $u($.$) and g($.$) (Kimball and Weil$ 2009).
} 
the inequality (8) reduces to

$$
\frac{u^{\prime \prime \prime}(w)}{-u^{\prime \prime}(w)} \leq 3 \frac{-u^{\prime \prime}(w)}{u^{\prime}(w)}+(1+m) \frac{u^{\prime}(w)}{u(w)} .
$$

Interestingly, this last inequality exhibits three different utility curvature coefficients, namely the familiar degrees of risk aversion and of prudence, as well as the reciprocal of the degree of fear of ruin $\frac{u}{u^{\prime}}$ (Foncel and Treich 2005). Take for instance a constant relative risk aversion (CRRA) utility function $u(w)=(1-\gamma)^{-1} w^{1-\gamma}$ with $\gamma \in(0,1)$. Then the condition (8) is equivalent to $\frac{m(1-\gamma)+\gamma}{w} \geq 0$, and is always satisfied under our parametric assumptions. ${ }^{15}$

We next discuss whether the comparison in condition (8) is robust to a change in the scaling of the utility function $u($.$) . This cannot be true$ generically. To see this, observe that the right hand side term of (8) depends directly on the function $u$ through $\frac{g^{\prime \prime \prime}(u(w))}{-g^{\prime \prime}(u(w))}$. As a result, this side of the equation can be arbitrarily affected by an additive change from $u($.$) to$ $u^{*}()=.u()+$.$b for some functions g($.$) , thus possibly modifying the sign$ of the inequality depending on the value of $b$. Note however that under a negative exponential SWF, i.e. $g(u)=-e^{-u}$, the term $\frac{g^{\prime \prime \prime}(u(w))}{-g^{\prime \prime}(u(w))}$ becomes a constant. Hence, our comparative statics results are not affected by an additive change in this case. This is not a surprise since it is well known that the ranking of prospects is not affected by such additive re-scaling under exponential SWFs (Bossert and Weymark 2004, Adler 2012). Consider alternatively an Atkinsonian SWF. Then the comparative statics analysis would not be affected by a "ratio-rescaling" of the utility function, namely by a multiplicative change from $u($.$) to u^{*}()=.a u($.$) with a>0$. To see this, observe that none of the curvature coefficients in (9) would be affected by such a multiplicative change. Again, this result is not surprising since the Atkinsonian function is known to be the only prioritarian SWF to display the ratio-rescaling invariance property (Bossert and Weymark 2004, Adler 2012).

Observe finally that (9) is more likely to be satisfied when the "inequity aversion" parameter $m$ increases. At the limit, when $m$ tends to infinity, i.e. for a Rawlsian-type SWF, the inequality (8) is always satisfied. This

\footnotetext{
${ }^{15}$ Observe that under our parameterization we get $u(0)=0$ so that $w=0$ can be interpreted as a minimal subsistence level of wealth. This "zeroing out" assumption (Adler 2012, Adler and Treich 2015) is not innocuous because the scaling of utility matters under prioritarianism.
} 
observation provides an intuition for the result. Indeed, under EPP and a Rawlsian-type SWF, the social planner's objective is to increase consumption of agent 2 in the worst state ex post (i.e., when $\widetilde{w}=w_{\text {inf }}$ ), as soon as the utility reached in that state is not higher than agent 1's utility. The social planner thus essentially chooses consumption such that $u(c) \approx u\left(w_{\text {inf }}-c\right)$. This tends to reduce the first agent's consumption compared to utilitarianism, given by $u^{\prime}(c)=E u^{\prime}(\widetilde{w}-c)$, and to reduce it even more compared to EAP (under a Rawlsian-type SWF), given by $u(c) \approx E u(\widetilde{w}-c)$.

\section{A simple model with learning}

In this section, we consider a specific three-agent model, and we will allow for the possibility of learning. The objective of the utilitarian social planner becomes

$$
\max _{c_{1}, c_{2}} u\left(c_{1}\right)+u\left(c_{2}\right)+E u\left(\widetilde{w}-c_{1}-c_{2}\right) .
$$

Note that perfect consumption smoothing across agents 1 and 2 is optimal here, i.e. $c_{1}=c_{2}=c$. The problem of finding optimal consumption levels then becomes

$$
c^{U}=\arg \max _{c} 2 u(c)+E u(\widetilde{w}-2 c) .
$$

Similarly, optimal consumption levels under EAP and EPP are defined by

$$
\begin{aligned}
c^{E A P} & =\arg \max _{c} 2 g(u(c))+g(E u(\widetilde{w}-2 c)), \\
c^{E P P} & =\arg \max _{c} 2 g(u(c))+E g(u(\widetilde{w}-2 c)) .
\end{aligned}
$$

It is easy to see then the comparison of $c^{U}, c^{E A P}$ and $c^{E P P}$ leads to the same results as in the Propositions before. Considering more (than 3 ) agents would not affect these results provided that perfect smoothing remains optimal across all agents except the last one who faces the risk. However, the situation becomes more complex if learning is allowed, as we now show. ${ }^{16}$

For simplicity, we assume perfect learning once the first consumption allocation decision has been made by the social planner. In other words, the consumption allocation problem between agents 2 and 3 is made under

\footnotetext{
${ }^{16}$ Learning is an important factor affecting risk policies in general, especially for long term problems. See for example the literature on climate change (Ulph and Ulph 1997 and Gollier, Jullien and Treich 2000).
} 
certainty, that is after the realization of $\widetilde{w}$ is known. This implies that perfect smoothing across agents 2 and 3 is optimal both under prioritarianism and under utilitarianism. Hence, before the uncertainty is revealed, the optimal (risky) consumption of agent 2 is equal to

$$
c_{2}^{*}=\frac{\widetilde{w}-c_{1}}{2} .
$$

Using obvious notations, the optimal consumption of the first agent for utilitarianism and EPP are then defined as follows (the EAP case is treated later)

$$
\begin{aligned}
c^{U L} & =\arg \max _{c} u(c)+2 E u\left(\frac{\widetilde{w}-c}{2}\right), \\
c^{E P P L} & =\arg \max _{c} g(u(c))+2 E g\left(u\left(\frac{\widetilde{w}-c}{2}\right)\right) .
\end{aligned}
$$

Note that the effect of learning under utilitarianism, and under ex post prioritarianism, is given by comparing $c^{U L}$ to $c^{U}$ in (10) and $c^{E P P L}$ to $c^{E P P}$ in (12). It is not very difficult to show that learning usually increases the first agent's consumption under utilitarianism and EPP compared to the no learning (i.e., "risk") case. ${ }^{17}$ The intuition is that learning allows to better smooth consumption in the future between agents 2 and 3, which thus increases the average future utility in the society, and in turn decreases the marginal utility of consumption (Epstein 1980, Eeckhoudt, Gollier and Treich 2005).

Assuming learning, we now want to compare consumption under utilitarianism and EPP, i.e. $c^{U L}$ and $c^{E P P L}$. This amounts to comparing consumption under $u($.$) and v()=.g(u()$.$) , and this comparison is immediate from$ previous Proposition 3. Indeed we can show that under learning, the first agent consumes less under EPP than under utilitarianism iff (8) holds.

The case of EAP is more difficult. Indeed, viewed from the initial consumption allocation problem, the future utility of agents 2 and 3 equals

\footnotetext{
${ }^{17}$ Let us first compare consumption under learning and under risk assuming utilitarianism. Under learning, the FOC is given by $u^{\prime}(c)-E u^{\prime}\left(\frac{\widetilde{w}-c}{2}\right)=0$, while under risk it is given by $u^{\prime}(c)-E u^{\prime}(\widetilde{w}-2 c)=0$. Thus there is more current consumption under learning iff $E u^{\prime}(\widetilde{w}-2 c) \geq E u^{\prime}\left(\frac{\widetilde{w}-c}{2}\right)$ given that $u^{\prime}(c)-E u^{\prime}(\widetilde{w}-2 c)=0$. Observe now $E u^{\prime}(\widetilde{w}-2 c)=\frac{1}{2} u^{\prime}(c)+\frac{1}{2} E u^{\prime}(\widetilde{w}-2 c) \geq E u^{\prime}\left(\frac{\widetilde{w}-c}{2}\right)$ by Jensen's inequality and $u^{\prime \prime \prime} \geq 0$. This leads to the result that given prudence learning increases early consumption under utilitarianism. Note then that the role of learning under EPP is similar by just replacing $u$ by $v=g(u)$ in the previous reasoning. But then observe that $g^{\prime \prime \prime} \geq 0$ ensures $v^{\prime \prime \prime} \geq 0$ so the result also carries over under $u^{\prime \prime \prime} \geq 0$ and $g^{\prime \prime \prime} \geq 0$.
} 
$u\left(\frac{\widetilde{w}-c}{2}\right)$ and is risky, which matters under EAP. Optimal consumption is given by

$$
c^{E A P L}=\arg \max _{c} g(u(c))+2 g\left(E u\left(\frac{\widetilde{w}-c}{2}\right)\right) .
$$

The problem here is that the EAP criterion is time-inconsistent (Broome 1984, Adler and Sanchirico 2006). Technically, this relates to the fact that the intertemporal utility function in (13) is not linear in probabilities (Hammond 1983, Epstein and Le Breton 1992). ${ }^{18}$ This means that, by contrast with utilitarianism or EPP, the dynamic optimization problem cannot be formulated recursively under EAP. To see that, consider the second stage problem. After the resolution of uncertainty, i.e. $\widetilde{w}=w$, the EAP social planner evaluates the wellbeing of agents 2 and 3 by $2 g\left(u\left(\frac{w-c}{2}\right)\right)$. Therefore, before the resolution of uncertainty, this social planner would be time-consistent by averaging future values of wellbeing across the possible states of the world, namely by considering $2 E g\left(u\left(\frac{\widetilde{w}-c}{2}\right)\right)$. But this does not correspond to the ex ante objective of the EAP social planner under learning, as defined in (13). ${ }^{19}$ This form of time-inconsistency can be seen as an important drawback of EAP approach.

Observe now that the comparison under learning of utilitarianism and (time-inconsistent) EAP is similar to this comparison without learning. DARA is, again, the instrumental condition on the utility function that drives the analysis. A sketch of the proof of this result follows. We want to compare $c^{E A P L}$ and $c^{U L}$. Respective FOCs equal $g^{\prime}(u(c)) u^{\prime}(c)-g^{\prime}\left(E u\left(\frac{\widetilde{w}-c}{2}\right)\right) E u^{\prime}\left(\frac{\widetilde{w}-c}{2}\right)=$ 0 and $u^{\prime}(c)-E u^{\prime}\left(\frac{\widetilde{w}-c}{2}\right)=0$. Therefore we are done if we can show $u(c) \leq$

\footnotetext{
${ }^{18}$ Note that this nonlinearity might also imply a negative value of information (Wakker 1988). But we can show that this is not the case here. Indeed the utility reached under learning (see (13)) is always higher than the one reached under no learning (see (11)) iff $g\left(E u\left(\frac{\widetilde{w}-c}{2}\right)\right) \geq \frac{1}{2}(g(u(c))+g(E u(\widetilde{w}-2 c))$. This inequality always holds under $g$ and $u$ concave by simply applying twice the Jensen's inequality.

${ }^{19}$ This observation is reminiscent of Myerson (1981)'s egalitarian-father example, showing that the evaluation of social welfare may depend on the timing of the resolution of uncertainty. The example goes as follows. An egalitarian father has two children, who can become clerk, teacher or doctor depending whether they go to college for respectively 0,4 or 8 years. The problem is that the father can afford only 8 years of college. Moreover, the father prefers that the two children have the same situation, whereas the children prefer a $50 \%$ chance of being clerk/doctor to being teacher for sure. Suppose that a fair coin decides who will go to the medical school. Before the coin toss, this randomization device Pareto-dominates the "both teachers" plan. Yet, after the coin toss, this device will seem unegalitarian and the father prefers the "both teachers" plan.
} 
$E u\left(\frac{\widetilde{w}-c}{2}\right)$ with $u^{\prime}(c)=E u^{\prime}\left(\frac{\widetilde{w}-c}{2}\right)$. By a similar reasoning as in the proof of Proposition 1, this holds iff DARA. We thus find that, under learning, the first agent consumes more under EAP than under utilitarianism iff $u($.$) is$ DARA.

Overall, these results under learning indicate that the first agent consumes less under EPP, and more under EAP, than under utilitarianism. To obtain these results, we need similar conditions as in the simple two-agent model without learning. Thus, we conclude that the results obtained in previous sections are robust to the introduction of learning.

\section{Other SWFs}

In this section, we widen the analysis by considering two additional families of SWFs: "transformed utilitarianism" and "transformed EPP" (Adler, Hammitt and Treich 2014). ${ }^{20}$ An important property is that the SWFs corresponding to these two cases are no longer separable across agents.

We consider again the basic two-agent model. Under transformed utilitarianism, the optimal consumption of the first agent is defined by

$$
c^{T U}=\arg \max _{c} E h[u(c)+u(\widetilde{w}-c)],
$$

in which $h$ is assumed to be strictly increasing and twice differentiable. Note that if $h$ is nonlinear, $c^{T U}$ does not coincide in general with the optimal consumption under plain utilitarianism, namely with $c^{U} \cdot{ }^{21}$ In particular, we have $c^{T U} \leq c^{U}$ iff

$$
E\left\{h^{\prime}\left[u\left(c^{U}\right)+u\left(\widetilde{w}-c^{U}\right)\right]\left(u^{\prime}\left(c^{U}\right)-u^{\prime}\left(\widetilde{w}-c^{U}\right)\right)\right\} \leq 0,
$$

or equivalently iff

$$
\operatorname{Cov}\left(h^{\prime}\left[u\left(c^{U}\right)+u\left(\widetilde{w}-c^{U}\right)\right], u^{\prime}\left(c^{U}\right)-u^{\prime}\left(\widetilde{w}-c^{U}\right)\right) \leq 0,
$$

since $E\left(u^{\prime}\left(c^{U}\right)-u^{\prime}\left(\widetilde{w}-c^{U}\right)\right)=0$ by the definition of $c^{U}$. Notice that the term $\left(u^{\prime}\left(c^{U}\right)-u^{\prime}\left(w-c^{U}\right)\right)$ is always increasing in $w$ while the term $h^{\prime}\left[u\left(c^{U}\right)+u\left(w-c^{U}\right)\right]$ is always decreasing in $w$ iff $h^{\prime}$ is decreasing. Therefore

\footnotetext{
${ }^{20}$ The "transformed EAP" case is equivalent to EAP.

${ }^{21}$ In the following, we assume that the second order condition is always satisfied. Note that this need not be the case if $h$ is "sufficiently" convex.
} 
the concavity of the transformation function $h$ is a necessary and sufficient condition for transformed utilitarianism to reduce agent 1's consumption compared to plain utilitarianism, i.e. $c^{T U} \leq c^{U}$.

Under transformed EPP, the optimal consumption of the first agent is defined by

$$
c^{T E P P}=\arg \max _{c} E h[g(u(c))+g(u(\widetilde{w}-c))] .
$$

Simply observe now that the function $v()=.g(u()$.$) is also strictly increas-$ ing and strictly concave under our assumptions on $g$. As a result, we can straightforwardly use the above reasoning to conclude that the concavity of the transformation function $h$ is also a necessary and sufficient condition for transformed EPP to reduce agent 1's consumption compared to plain EPP, i.e. $c^{T E P P} \leq c^{E P P}$. Moreover, we know from the analysis above that agent 1 's consumption is lower under EPP than under utilitarianism iff the condition (8) is satisfied. We can therefore conclude that EPP, either under the plain version or under the transformed version with $h$ concave, leads to less consumption for agent 1 compared to utilitarianism under that same condition (8), i.e. $c^{T E P P} \leq c^{U}$.

The previous observation suggests however that the comparison between agent 1's consumption under utilitarianism and under transformed EPP is not clear when $h$ is convex. A case in point is the prioritarian case of Fleurbaey (2010)'s EDE. The optimal consumption of the first agent under EDE is defined by

$$
c^{E D E}=\arg \max _{c} E g^{-1}\left(\frac{1}{2} g(u(c))+\frac{1}{2} g(u(\widetilde{w}-c))\right),
$$

so that $h(x)=g^{-1}(x / 2)$ is strictly convex since $g$ is strictly concave. Following the above observations, we know that $c^{E D E}$ is greater than $c^{E P P}$, which is also greater than $c^{T E P P}$ with any $h$ concave. However, we cannot use previous results to directly compare $c^{E D E}$ to $c^{U}$. Under EDE, the FOC is given by

$$
E \frac{g^{\prime}(u(c)) u^{\prime}(c)-g^{\prime}(u(\widetilde{w}-c)) u^{\prime}(\widetilde{w}-c)}{g^{\prime}\left(g^{-1}\left(\frac{1}{2} g(u(c))+\frac{1}{2} g(u(\widetilde{w}-c))\right)\right)}=0 .
$$

Using (15), we derive in the appendix the necessary and sufficient condition to compare $c^{E D E}$ and $c^{U}$ when $\widetilde{w}$ is "small" in the sense of a second order approximation. This condition takes the form of an inequality which is always equal to zero under Atkinsonian and CRRA utility functions. In other words, we have $c^{E D E}=c^{U}$ under this set of assumptions. This suggests that this 
special type of ex post approach introduced by Fleurbaey (2010) can be viewed as a limit case of "transformed EPP" leading to the same consumption level as under utilitarianism.

\section{Conclusion}

In this paper, we have considered a simple cake eating problem under risk. The social planner must decide how much of the cake is consumed by a first agent, given that the remaining portion of the cake left for a second agent is unknown. The social planner may be utilitarian, ex ante prioritarian or ex post prioritarian. We have shown that, under standard assumptions on utility and social welfare functions, prioritarianism always leads to more consumption by the first agent under an ex ante approach, but to less consumption under an ex post approach, than under utilitarianism. These standard assumptions include the familiar constant relative risk aversion utility and Atkinsonian social welfare functions. Moreover, in an extension to "transformed" ex post settings, we have exhibited a simple condition so that there is also less consumption under the ex post prioritarian approach than under utilitarianism.

Why is this result interesting? Many economic problems combine an equity, time and risk dimension. Consider the general idea that the risk of future climate change justifies less consumption of energy today. The traditional argument in the economics literature relies on a precautionary savings motive applied to a risky social situation. Our paper shows that this argument is reinforced by a moral prioritarian argument only under the ex post approach, but is weakened under the ex ante approach. More generally, the paper shows that the way one applies equity considerations to conditions of risk sensitively matters for economic policies.

We conclude the paper by outlining a few possible directions for future research. Our consumption model is parsimonious, but too restrictive to capture some essential features of real-world policy applications like climate change. In particular, the model does not capture cumulative pollution ef-

fects. Moreover, it considers an additive risk. Yet, in many applications the future risk grows with the accumulation of past consumption, and is thus multiplicative. Another ambitious extension would be to consider the general problem of a social planner who must decide transfers between agents facing different risks. Indeed, in our model, we have considered an extreme 
situation in which only one agent faces a risk. Yet, such an extension to multiple risks is complex and technically challenging, as we already noted in the text. Finally, another research direction relates to our assumption that individual preferences are homogeneous. Although controversial in the social choice literature, this assumption is prevalent in applied welfare economics. For instance, essentially all the literature on climate change that we are aware of assumes homogeneous utilities. Yet, it seems reasonable to allow for the possibility that the preferences of future generations may differ from ours. It would seem interesting to explore the impact of this difference in future preferences on intergenerational allocation decisions.

\section{Appendix: The EDE case}

In this appendix, we derive conditions so that consumption under Fleurbaey (2010)'s equally distributed equivalent (EDE) is lower than under utilitarianism. Formally, using the FOCs (2) and (15), we want to show

$$
u^{\prime}(c)-E u^{\prime}(\widetilde{w}-c)=0 \Longrightarrow E \frac{g^{\prime}(u(c)) u^{\prime}(c)-g^{\prime}(u(\widetilde{w}-c)) u^{\prime}(\widetilde{w}-c)}{g^{\prime}\left(g^{-1}\left(\frac{1}{2} g(u(c))+\frac{1}{2} g(u(\widetilde{w}-c))\right)\right)} \leq 0 .
$$

We use the diffidence theorem (Gollier 2001, page 86-87). More precisely, we use a Lemma which is directly based on a necessity part of the diffidence theorem when applied to "small risks" in the sense of a second-order approximation.

Lemma. (Gollier 2001) Let the problem:

$$
\text { for all } \widetilde{w}, E f_{1}(\widetilde{w})=0 \Longrightarrow E f_{2}(\widetilde{w}) \leq 0 \text {. }
$$

Assume that there exists a scalar $w_{0}$ such that $f_{1}\left(w_{0}\right)=f_{2}\left(w_{0}\right)=0$ with $f_{1}^{\prime}\left(w_{0}\right) \neq 0$. Then, a necessary and sufficient for (17) for any "small" $\widetilde{w}$ around $w_{0}$ is

$$
f_{2}^{\prime \prime}\left(w_{0}\right) \leq \frac{f_{2}^{\prime}\left(w_{0}\right)}{f_{1}^{\prime}\left(w_{0}\right)} f_{1}^{\prime \prime}\left(w_{0}\right) .
$$

To study condition (16), we now simply apply this Lemma with $f_{1}(w)=$ $u^{\prime}(c)-u^{\prime}(w-c)$ and

$$
f_{2}(w)=\frac{g^{\prime}(u(c)) u^{\prime}(c)-g^{\prime}(u(w-c)) u^{\prime}(w-c)}{g^{\prime}\left(g^{-1}\left(\frac{1}{2} g(u(c))+\frac{1}{2} g(u(w-c))\right)\right)} .
$$


Observe that under $w_{0}=2 c$, we have $f_{1}\left(w_{0}\right)=f_{2}\left(w_{0}\right)=0$. We then easily obtain $f_{1}^{\prime}(2 c)=-u^{\prime \prime}(c) \neq 0$ and $f_{1}^{\prime \prime}(2 c)=-u^{\prime \prime \prime}(c)$. Moreover, we can compute

$$
f_{2}^{\prime}(2 c)=-\frac{u^{\prime}(c)^{2} g^{\prime \prime}(u(c))}{g^{\prime}(u(c))}-u^{\prime \prime}(c)>0,
$$

and

$$
\begin{aligned}
f_{2}^{\prime \prime}(2 c)= & g^{\prime}(u(c))^{-2}\left[-2 g^{\prime}(u(c)) u^{\prime}(c) g^{\prime \prime}(u(c)) u^{\prime \prime}(c)+u^{\prime}(c)^{3}\left(g^{\prime \prime}(u(c))^{2}\right.\right. \\
& \left.\left.-g^{\prime}(u(c)) g^{\prime \prime \prime}(u(c))\right)-g^{\prime}(u(c))^{2} u^{\prime \prime \prime}(c)\right] .
\end{aligned}
$$

Assuming $g(u)=(1-m)^{-1} u^{1-m}$ with $u>0$ and $m>0$, we obtain

$\frac{f_{2}^{\prime \prime}(2 c)}{f_{2}^{\prime}(2 c)}-\frac{f_{1}^{\prime \prime}(2 c)}{f_{1}^{\prime}(2 c)}=\frac{m u^{\prime}(c)^{4}}{u(c) u^{\prime \prime}(c)\left(-m u^{\prime}(c)^{2}+u(c) u^{\prime \prime}(c)\right)}\left[\frac{u^{\prime \prime}(c)}{u^{\prime}(c)}-2 \frac{u(c)}{u^{\prime}(c)} \frac{u^{\prime \prime}(c)^{2}}{u^{\prime}(c)^{2}}+\frac{u(c)}{u^{\prime}(c)} \frac{u^{\prime \prime \prime}(c)}{u^{\prime}(c)}\right]$.

Note that the sign of this last expression only depends on the utility function $u($.$) through the term in brackets. This term can be respectively positive$ or negative for some utility functions (e.g., take respectively $u(c)=c+\sqrt{c}$ and $u(c)=1-1 /(1+c))$. However, if we assume $u(w)=(1-\gamma)^{-1} w^{1-\gamma}$ with $\gamma \in(0,1)$, it is immediate that the term in bracket is always equal to zero. Therefore, the necessary and sufficient condition (18) provided by the Lemma above is always satisfied "just" under Atkinsonian and CRRA functions.

Using the same reasoning as above, one can now simply compare $c^{E P P}$ to $c^{U}$. To do so, it is enough to use the Lemma above with $f_{1}(w)=u^{\prime}(c)-$ $u^{\prime}(w-c)$ and $f_{2}(w)=g^{\prime}(u(c)) u^{\prime}(c)-g^{\prime}(u(w-c)) u^{\prime}(w-c)$, consistent with (7). Assuming Atkinsonian and CRRA functions, $\frac{f_{2}^{\prime \prime}(2 c)}{f_{2}^{\prime}(2 c)}-\frac{f_{1}^{\prime \prime}(2 c)}{f_{1}^{\prime}(2 c)}$ then simplifies to $\frac{m(\gamma-1)}{c}$, which is always strictly negative. This shows that under "small" risks, and under Atkinsonian and CRRA functions, while we have $c^{E D E}=c^{U}$, we nevertheless still have $c^{E P P}<c^{U}$. This is consistent with the idea that EDE can be viewed as a limit case of "transformed EPP" leading to the same consumption level as under utilitarianism. 


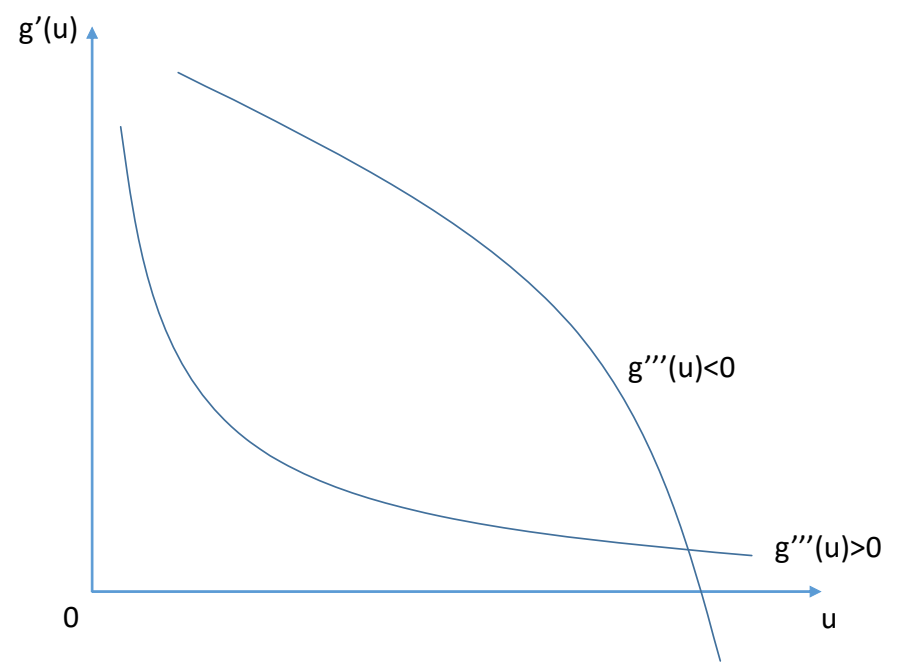

Figure 1:

This figure illustrates that the conditions $g^{\prime}(u)>0, g^{\prime \prime}(u)<0$ together with $g^{\prime \prime \prime}(u)<0$ for all $u>0$ are mutually inconsistent. 


\section{References}

Adler, M.D., 2012, Well-Being and Fair Distribution: Beyond Cost-Benefit Analysis, Oxford University Press.

Adler, M.D., 2016a, Benefit-cost analysis and distributional weights: An overview, Review of Environmental Economics and Policy 10, 264-285.

Adler, M.D., 2016b, Extended preferences, chapter 17 in Oxford Handbook of Well-Being and Public Policy, Matthew Adler and Marc Fleurbaey eds., Oxford University Press, 476-517.

Adler, M.D., J.K. Hammitt and N. Treich, 2014, The social value of mortality risk reduction: VSL vs. the social welfare function approach, Journal of Health Economics 35, 82-93.

Adler, M.D. and C.W. Sanchirico, 2006, Inequality and uncertainty: Theory and legal applications, University of Pennsylvannia Law Review 155, 279-377.

Adler, M.D. and N. Treich, 2015, Prioritarianism and climate change, Environmental and Resource Economics 62, 279-308.

Ambec, S. and Y. Sprumont, 2002, Sharing a river, Journal of Economic Theory 107, 453-62.

Arrow, K.J., 1951, Social Choice and Individual Values, New York: John Wiley and Sons.

Bommier, A, Chassagnon A. and F. Le Grand, 2012, Comparative risk aversion: A formal approach with applications to saving behavior, Journal of Economic Theory 147, 1614-41.

Bommier, A., Lanz B. and S. Zuber, 2015, Models-as-usual for unusual risks? On the value of catastrophic climate change, Journal of Environmental Economics and Management 74, 1-22.

Bommier, A. and S. Zuber, 2008, Can preferences for catastrophe avoidance reconcile social discounting with intergenerational equity?, Social Choice and Welfare 31, 415-434.

Bossert W. and J.A. Weymark, 2004, Utility in social choice, Handbook of Utility Theory 2, 1099-1177, Boston: Kluwer Academic.

Broome, J., 1984, Uncertainty and fairness, Economic Journal 94, 624-32.

Broome, J., 1991, Weighing Goods: Equality, Uncertainty and Time. Wiley.

Dasgupta, P., 2008, Discounting climate change, Journal of Risk and Uncertainty 37, 141-69. 
Dhillon, A. and J.-F. Mertens, 1999, Relative utilitarianism, Econometrica $67,471-98$.

Diamond P.A., 1967, Cardinal welfare, individualistic ethics, and interpersonal comparison of utility: Comment, Journal of Political Economy 75, $765-66$.

Dietz S. and G.B. Asheim, 2012, Climate policy under sustainable discounted utilitarianism, Journal of Environmental Economics and Management 63, 321-335.

Drèze, J.H. and F. Modigliani, 1972, Consumption decisions under uncertainty, Journal of Economic Theory 5, 308-35.

Drèze J. and N. Stern, 1987, The theory of cost-benefit analysis, Handbook of Public Economics, volume II, Chapter 14, Elsevier (North-Holland).

Eeckhoudt L. and H. Schlesinger, 1994, A precautionary tale of risk aversion and prudence, in Models and Experiments in Risk and Rationality (eds. Munier B. and M. Machina), Kluwer Academic Publishers.

Eeckhoudt, L. and H. Schlesinger, 2006, Putting risk in its proper place, American Economic Review 96, 280-289.

Eeckhoudt, L., Gollier C. and N. Treich, 2005, Optimal consumption and the timing of the resolution of uncertainty, European Economic Review 49, 761-73.

Epstein, L.G., 1980, Decision-making and the temporal resolution of uncertainty, International Economic Review 21, 269-284.

Epstein, L.G. and M. Le Breton, 1993, Dynamically consistent beliefs must be Bayesian, Journal of Economic Theory 61, 1-22.

Epstein, L.G. and U. Segal, 1991, Quadratic social welfare functions, Journal of Political Economy 100, 691-712.

Epstein, L.G. and S.E. Zin, 1989, Substitution, risk aversion, and the temporal behavior of consumption and asset returns: A theoretical framework, Econometrica 57, 937-69.

Ferranna, M., 2016a, Fairness in cost benefit analysis: Equity-enhanced mean variance rules, mimeo, Toulouse School of Economics.

Ferranna, M., 2016b, Fairness, risk and the social cost of carbon, mimeo, Toulouse School of Economics.

Fleurbaey M., 2010, Assessing risky social situations, Journal of Political Economy 118, 649-80.

Fleurbaey M. and L. Bovens, 2012, Evaluating life or death prospects, Economics and Philosophy 28, 217-249. 
Fleurbaey M. and S. Zuber, 2015, Discounting, risk and inequality: A general approach, Journal of Public Economics 128, 34-49.

Foncel J. and N. Treich, 2005, Fear of ruin, Journal of Risk and Uncertainty $31,289-300$.

Gilbert R.J., 1979, Optimal depletion of an uncertain stock, Review of Economic Studies 46, 47-57.

Gollier C., 2001, The Economics of Risk and Time, The MIT Press.

Gollier C., 2003, Discounting an uncertain future, Journal of Public Economics 85, 149-66.

Gollier C., 2012, Pricing the Planet's Future: The Economics of Discounting in an Uncertain World, Princeton University Press.

Gollier C., Jullien B. and N. Treich, 2000, Scientific progress and irreversibility: An economic interpretation of the precautionary principle, Journal of Public Economics 75, 229-53.

Grant S., Kajii A., Pollack B. and S. Safra, 2010, Generalized utilitarianism and Harsanyi's impartial observer theorem, Econometrica 78, 1939-71.

Ha Duong M. and N. Treich, 2004, Risk-aversion, intergenerational equity and climate change, Environmental and Resource Economics 28, 195-207.

Hammond P., 1983, Ex-post optimality as a dynamically consistent objective for collective choice under uncertainty, Social Choice and Welfare: Symposium on collective choices, Amsterdam: North-Holland-Publication.

Harsanyi, J.C., 1975, Nonlinear social welfare functions, Theory and Decision 6, 311-32.

Holtug, N., 2010. Persons, Interests, and Justice. Oxford: Oxford University Press.

Jensen, S. and C.P. Traeger, 2014, Optimal climate change mitigation under long-term growth uncertainty: Stochastic integrated assessment and analytic findings, European Economic Review 69, 104-25.

Johansson-Stenman, O., 2005, Distributional weights in cost-benefit analysis - Should we forget about them?, Land Economics 81, 337-52.

Kaplow L., 2008, The Theory of Taxation and Public Economics, Princeton University Press.

Kemp, M., 1976, How to eat a cake of unknown size, Chapter 23 in Kemp, M., Three Topics in the Theory of International Trade (Amsterdam: North-Holland).

Khilstrom, R.E. and L.J. Mirman, 1974, Risk aversion with many commodities, Journal of Economic Theory 8, 361-388. 
Kimball M.S., 1990, Precautionary savings in the small and in the large, Econometrica 58, 53-73.

Kimball, M.S. and P. Weil, 2009, Precautionary saving and consumption smoothing across time and possibilities, Journal of Money, Credit and Banking 41, 245-84.

Lange A. and N. Treich, 2008, Uncertainty, learning and ambiguity in climate policy: Some classical results and new directions, Climatic Change 89, 7-21.

Leland, H.E., 1968, Saving and uncertainty: The precautionary demand for saving, Quarterly Journal of Economics 82, 465-73.

Loury, G.C., 1978, The optimal exploitation of an unknown reserve, Review of Economic Studies 45, 621-36.

Menegatti, M., 2001, On the conditions for precautionary saving, Journal of Economic Theory 98, 189-193.

Millner, A., 2013, On welfare frameworks and catastrophic climate risks, Journal of Environmental Economics and Management 65, 310-325.

Mongin, P. and M. Pivato, Social evaluation under risk and uncertainty, chapter 24 in Oxford Handbook of Well-Being and Public Policy, Matthew Adler and Marc Fleurbaey eds., Oxford University Press, 711-743.

Myerson, R.B., 1981, Utilitarianism, egalitarianism and the timing effect in social choice problems, Econometrica 49, 883-97.

Nagel, T., 1995, Equality and Partiality, Oxford University Press.

Nordhaus, W.D. 2008. A Question of Balance: Weighing the Options on Global Warming Policies. New Haven: Yale University Press.

Parfit, D., 1991, Equality or Priority, Lindley Lecture, University of Kansas. Reprinted in The Ideal of Equality (eds: M. Clayton and A. Williams), Houndmills: Palgrave 2000, 81-125.

Porter, T., 2012, In defence of the priority view, Utilitas 24, 197-206.

Roemer, J.E., 2008, The ethics of intertemporal distribution in a warming planet, Environmental and Resource Economics 48, 363-390.

Sandmo A., 1970, The effect of uncertainty on saving decisions, Review of Economic Studies 37, 353-60.

Selden, L., 1978, A new representation theorem over "certain $\times$ uncertain" consumption pairs: The "ordinal certainty equivalent" hypothesis, Econometrica 46, 1045-60.

Sen, A.K., 1970, Collective Choice and Social Welfare, Holden-Day: San Francisco. 
Stern, N., 2007, The Economics of Climate Change: The Stern Review. Cambridge: Cambridge University Press.

Traeger, C., 2012, Once upon a time preference - How rationality and risk aversion change the rationale for discounting, mimeo.

Tungodden, B., 2003, The value of equality. Economics and Philosophy 19, 1-44.

Ulph, A., 1982. The role of ex ante and ex post decisions in the valuation of life, Journal of Public Economics 18, 265-76.

Ulph, A. and D. Ulph, 1997, Global warming, irreversibility and learning, Economic Journal 107, 636-650.

Wakker, P.W., 1988, Nonexpected utility as aversion of information, Journal of Behavioral Decision Making 1, 169-75.

Weitzman, M.L., 2009, On modeling and interpreting the economics of catastrophic climate change, Review of Economics and Statistics 91, 1-19. 